\title{
A Simple Model of Sheet Metal Assembly*
}

Kathleen Hoffman ${ }^{\dagger}$ Fadil Santosa $a^{\ddagger}$

Abstract. This paper is meant to serve as a case study of mathematical modeling in industry. The problem, which arises in the automotive industry, is to predict the variation in the final assembly given the variation in the parts and tooling. In this paper, we present a simple model of welding and clamping of beams which demonstrates that the order in which the clamps and welds are applied influences the final shape of the assembly.

The modeling of the process is done by simple mechanics. To solve the mathematical problem, we use standard ideas from constrained optimization and scientific computation. Additionally, using a statistical simulation we show that clamping and welding from the inside out leads to a smaller standard deviation in the result (as measured by the displacement of the right end of the beams) in response to normal distributions of variations in parts and welding. The findings help explain why certain welding sequences may be preferred.

Key words. modeling, sheet metal assembly, constrained optimization, joining, welding, simulation

AMS subject classifications. $74 \mathrm{~B} 05,65 \mathrm{~K} 10,81 \mathrm{~T} 80$

DOI. $10.1137 / \mathrm{S} 0036144501382307$

I. Introduction. Many problems arising in industrial settings are amenable to mathematical formulation and solution using techniques available to upper level undergraduates. In this paper, we present an example of such a problem that arose in the automobile manufacturing industry.

Assembling sheets of metal using clamps and spot welds is an important part of the manufacturing process in the automotive industry. For instance, a door of a car consists of several pieces of stamped sheet metals which are welded together to produce a strong structure. After thousands of clamps and welds, the final assembled product, the door, must be within certain specified tolerances in order to fit properly. The variation in the final product is influenced by the variations in parts and the assembly procedure. Our goal is to use modeling and simulation to predict the effect of variations of the parts and welding on the variation of the final product.

In particular, observations on the assembly-line floor led to the conclusion that the order in which the sequence of clamps and spot welds are applied affects the

*Received by the editors October 17, 2001; accepted for publication (in revised form) November 22, 2002; published electronically August 11, 2003. This work was supported by the Institute for Mathematics and Its Applications (IMA), which receives its major funding from the Division of Mathematical Sciences of the National Science Foundation.

http://www.siam.org/journals/sirev/45-3/38230.html

$\dagger$ Department of Mathematics and Statistics, University of Maryland, Baltimore County, Baltimore, MD 21250 (khoffman@math.umbc.edu).

${ }^{\ddagger}$ School of Mathematics, University of Minnesota, Minneapolis, MN 55455 (santosa@math. umn.edu). 

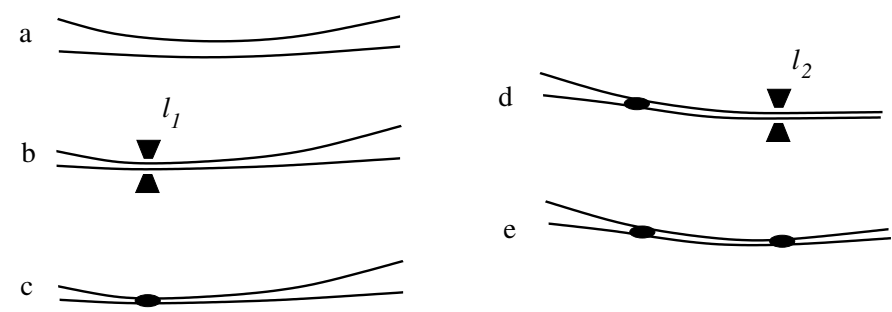

Fig. I In (a) two sheets of slightly different shape are to be joined. A clamp is applied at location $l_{1}$ in (b), and the two pieces are welded there. In (c) the clamp has been released. Another clamp is applied at location $l_{2}$, and another weld is made at that location as shown in (d). The final joined product is displayed in (e).

final shape of the product. Specifically, for illustrative purposes consider two sheets of metal that must first be clamped and then welded at two specific locations $l_{1}$ and $l_{2}$, as shown in Figure 1a. First, the sheets of metal are clamped at $l_{1}$ (Figure 1b), welded together at the clamped location, and the clamps removed (Figure 1c). Now the sheets of metal are fixed relative to each other at the welding location. A second clamp is now applied at the second location $l_{2}$ (Figure 1d) and then the sheets are welded together a second time (Figure 1e). Suppose now we reverse the welding sequence; i.e., with the same two sheets, first clamp the two sheets at location $l_{2}$, weld the sheets together, and release the clamp. Then, clamp the two sheets at location $l_{1}$, weld the sheets together, and release the clamp. The final shape of the sheets of metal that were first clamped and welded at $l_{1}$ followed by $l_{2}$ is not the same as the final shape of the sheets of metal that were first clamped at $l_{2}$ followed by $l_{1}$. That is, the order in which the clamps and spot welds are applied affects the shape of the final product.

Although this sequence dependence has been demonstrated by direct simulation using commercial finite element simulation software $[1,2,3,4]$, it was felt that they do not provide sufficient insight into the causes contributing to the observed sequence dependence. Our goal is to develop a simple model of a clamping and spot welding procedure that demonstrates and explains this sequence dependence. Our first simplifying assumption is to try to reproduce this sequence dependence in the clamping and welding procedure with a model of two one-dimensional beams rather than two twodimensional sheets of metal. This assumption simplifies the necessary computations and facilitates understanding of the actual sequence dependence without unnecessary complications of a two-dimensional model. What we discover in studying the onedimensional beam model is that nonlinearity in the model is the main cause, and the way in which it affects sequence dependence can be identified. Additionally, we use statistical simulation to determine the order of clamping and welding that leads to the least amount of variation in the final product.

This problem is ideally suited for an upper level undergraduate class in modeling. It is of contemporary interest to the manufacturing community and uses a variety of standard techniques to provide an intuitive explanation of a known phenomenon. The beam model is formulated as a series of rigid links allowing both theoretical and numerical linear programming techniques to be used in its solution. The solution of this problem requires theoretical ideas from mechanics, constrained optimization, and linearity to be combined with numerical techniques ranging from computing constrained optima to random variable generation and statistical simulations. 

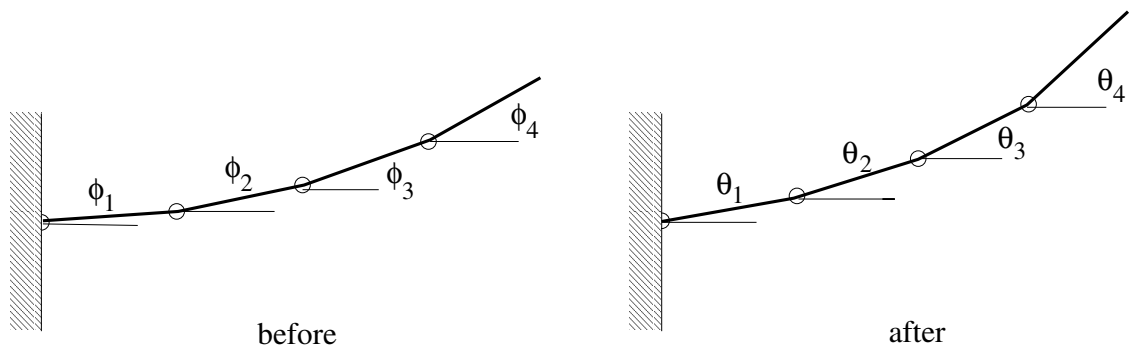

Fig. 2 A diagram depicting a simplified beam model. Each link is rigid, and a torsional spring is attached at each connection. The beam can have an initial shape as described by the angles $\phi_{1}, \phi_{2}, \ldots, \phi_{n}$. The beam configuration after deformation is completely described by the angles $\theta_{1}, \theta_{2}, \ldots, \theta_{n}$.

Modeling arises in developing the model of the beam as well as the clamping and spot welding conditions. A simplified model of rigid links connected by springs is presented as the variational formulation of Hooke's law with added complexity due to the nonzero initial bending angles of the links. The model that does not exhibit sequence dependence can be solved analytically, but the nonlinearity added to the model that leads to the sequence dependence is solved numerically using a MATLAB routine for constrained optimization. Finally, a Monte Carlo simulation that brings in the statistical ideas of normal distribution, mean, and standard deviation is used to demonstrate which sequence of clamps and spot welds is best.

The problem is presented in section 2. The linear model that does not exhibit the sequence dependence is presented in section 3. The nonlinear model that does demonstrate the sequence dependence is described in section 4 . Section 5 contains the results of the simulations that statistically demonstrate the order of clamping and welding that produces the least amount of total variation in the final product. Conclusions are discussed in section 6. Possible exercises or discussion points are suggested.

2. Problem Formulation. The process that we wish to model involves first clamping two beams at a specified location with a specified slope, then applying a spot weld that maintains the relative position of the two beams through all future manipulations of the beams. In order to elicit information regarding sequence dependence, we will first clamp, then spot weld the two beams in two different locations, $l_{1}=\left(x_{1}, y_{1}\right)$ and $l_{2}=\left(x_{2}, y_{2}\right)$ with $x_{1}<x_{2}$. The clamp and spot weld will first be applied to location $l_{1}$, then $l_{2}$, and, in a different simulation, the clamp and spot weld will first be applied to location $l_{2}$ followed by $l_{1}$. The final shape of the two simulations will determine if the model demonstrates the sequence dependence observed on the assembly-line floor.

Each beam is modeled as a series of $n$ rigid links, each with unit length. The links are connected with torsional springs with constant $K$. The left end of the beam is attached to the wall also with a spring of constant $K$ (see Figure 2). The beam resists bending by the torques that occur at the connections. The initial shape of the beam is prescribed by the angles $\phi_{1}, \phi_{2}, \ldots, \phi_{n}$. If the angles after deformation are $\theta_{1}, \theta_{2}, \ldots, \theta_{n}$, then the energy stored in the beam (assuming linear springs and small 
angular changes) is

$$
E=\frac{1}{2} K\left(\theta_{1}-\phi_{1}\right)^{2}+\sum_{j=2}^{n} \frac{1}{2} K\left(\theta_{j}-\phi_{j}-\theta_{j-1}+\phi_{j-1}\right)^{2} .
$$

This form assumes that the right end of the beam is free. Equilibrium solutions of the force balance laws correspond to critical points of the energy function. In order to determine the stable configurations, we seek an equilibrium deformation that will minimize this energy.

This simplified model is necessary because of the computational complexity of the problem. If we simply discretize a nonlinear beam, the computations become unwieldy due to the nonlinear constraints that model the clamping and welding conditions. The simplified model was sufficient to achieve our goal of explaining the sequence dependence that arises in the clamping and welding procedure.

3. Model without Sequence Dependence. If we take the point of view that displacement of the beam is small in the sense to be described, we arrive at a model of welding which does not depend on the order in which the welds are applied. While the model takes into consideration the initial shape of the beams, the displacements are supposed to be small, resulting in no $x$-displacements of the beam. Therefore a beam's displacement is completely described by the $y$-displacement of the nodes.

To arrive at this model, suppose that the current beam position is described by the angles $\theta_{1}, \theta_{2}, \theta_{3}, \ldots, \theta_{n}$. Let the positions of the nodes (joints) in the links be given by $\left(x_{i}, y_{i}\right), i=1, \ldots, n+1$; then

$$
\begin{aligned}
& x_{1}=0, \quad x_{i}=\sum_{j=1}^{i-1} \cos \theta_{j}, \quad i=2, \ldots, n+1, \\
& y_{1}=0, \quad y_{i}=\sum_{j=1}^{i-1} \sin \theta_{j}, \quad i=2, \ldots, n+1 .
\end{aligned}
$$

Let $\Delta \theta_{i}=\theta_{i}-\phi_{i}$, and assume

(A) that the initial shape of the beam is described by the small angles $\Delta \theta_{i}=$ $O\left(\varepsilon^{2}\right)$, where $\varepsilon \ll 1$.

Under this assumption,

$$
\begin{aligned}
\cos \theta_{i} & =\cos \left(\phi_{i}+\Delta \theta_{i}\right) \\
& =\cos \phi_{i} \cos \Delta \theta_{i}-\sin \phi_{i} \sin \Delta \theta_{i} \\
& \approx \cos \phi_{i}+O\left(\varepsilon^{3}\right) .
\end{aligned}
$$

Similarly,

$$
\begin{aligned}
\sin \theta_{i} & =\sin \left(\phi_{i}+\Delta \theta_{i}\right) \\
& \approx \sin \phi_{i}+\Delta \theta_{i}+O\left(\varepsilon^{3}\right) .
\end{aligned}
$$

This simplification reminds us of the model for a beam of small initial angles $\phi_{i}$ found in Washizu [6], where the small angle assumption for the initial shape of the beam is appropriately replaced with a small curvature assumption. These results, when 
before clamping

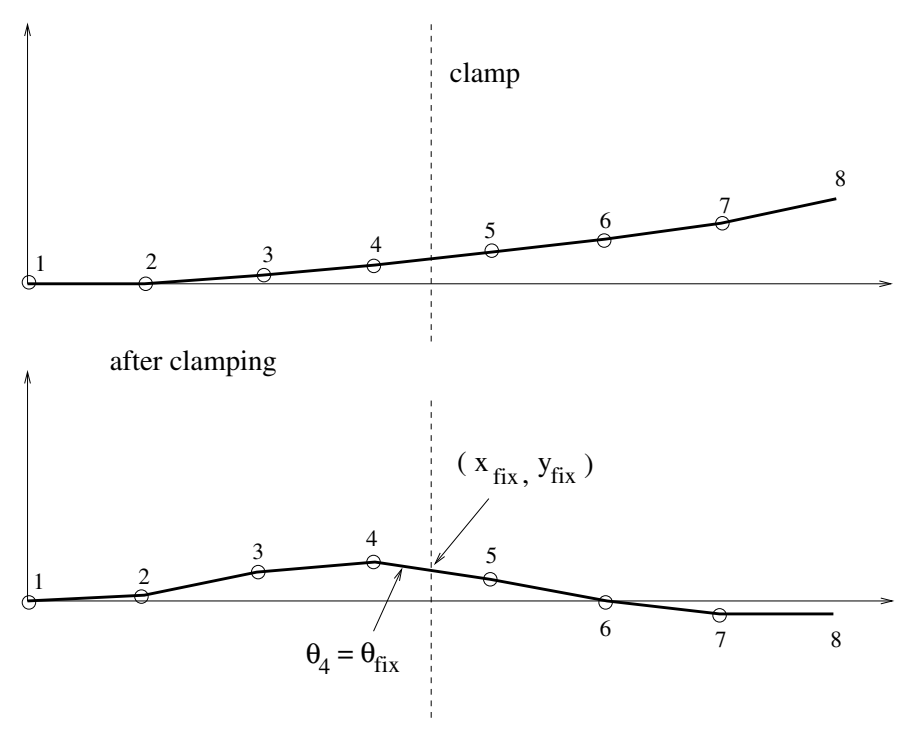

Fig. 3 In the seven-link beam, we assume that the clamp is applied on the fourth link. The clamp has the effect of forcing a point on that link to be at $\left(x_{\mathrm{fix}}, y_{\mathrm{fix}}\right)$. Moreover, the angle of the fourth link is prescribed by the clamp to be at $\theta_{\text {fix }}$.

substituted in the expression for node location in (3.1), imply that

$$
\begin{aligned}
x_{i} & \approx \sum_{j=1}^{i-1} \cos \phi_{j}, \quad i=2, \ldots, n+1, \\
y_{i} & \approx \sum_{j=1}^{i-1}\left(\sin \phi_{j}+\Delta \theta_{j}\right), \quad i=2, \ldots, n+1 .
\end{aligned}
$$

We see in (3.2) that the $x$-location of the nodes are fixed, whereas their $y$-locations are linear in the angular change $\Delta \theta_{i}$.

3.I. Clamping. We model a clamp by requiring a link to go through a point $\left(x_{\mathrm{fix}}, y_{\mathrm{fix}}\right)$ at a specified angle $\theta_{\mathrm{fix}}$. Consider a beam consisting of seven links. Suppose that a clamp is applied to link 4 as shown in Figure 3. The conditions that must be met are

$$
\begin{aligned}
& x_{4}=x_{\mathrm{fix}}+t \cos \theta_{4}, \\
& y_{4}=y_{\mathrm{fix}}+t \sin \theta_{4}, \\
& \theta_{4}=\theta_{\mathrm{fix}},
\end{aligned}
$$

where $t$ essentially defines the signed distance along the link 4 , the link in which the clamp is applied. Since $x_{4}$ is basically "static" and $\cos \theta_{4} \approx \cos \phi_{4}$, we use (3.3a) to solve for $t$ :

$$
t=\frac{\cos \phi_{1}+\cos \phi_{2}+\cos \phi_{3}-x_{\mathrm{fix}}}{\cos \phi_{4}}
$$


It is worth noting that the determination of $t$ is done entirely from the initial geometry under assumption (A). In particular, it is independent of the angular displacements.

The constraint (3.3b), under assumption (A), becomes

$$
y_{4} \approx y_{\mathrm{fix}}+t \sin \phi_{4}+t \Delta \theta_{4} .
$$

Using (3.2b) to determine the expression for $y_{4}$, we can write this constraint as

$$
\sum_{i=1}^{3} \Delta \theta_{i}-t \Delta \theta_{4}=y_{\text {fix }}+t \sin \phi_{4}-\sin \phi_{1}-\sin \phi_{2}-\sin \phi_{3}:=F .
$$

Condition (3.3c) simply states that

$$
\Delta \theta_{4}=\theta_{\text {fix }}-\phi_{4}
$$

Viewing the angles as a vector

$$
\Delta \boldsymbol{\theta}=\left(\begin{array}{lllllll}
\Delta \theta_{1} & \Delta \theta_{2} & \Delta \theta_{3} & \Delta \theta_{4} & \Delta \theta_{5} & \Delta \theta_{6} & \Delta \theta_{7}
\end{array}\right)^{T}
$$

and letting

$$
c_{1}^{T}=\left(\begin{array}{lllllll}
1 & 1 & 1 & -t & 0 & 0 & 0
\end{array}\right)
$$

to reflect (3.5a), and

$$
c_{2}^{T}=\left(\begin{array}{lllllll}
0 & 0 & 0 & 1 & 0 & 0 & 0
\end{array}\right)
$$

to reflect (3.5b), we can rewrite (3.5a) and (3.5b) as

$$
\begin{gathered}
c_{1}^{T} \Delta \boldsymbol{\theta}-F=0, \\
c_{2}^{T} \Delta \boldsymbol{\theta}-\left(\theta_{\mathrm{fix}}-\phi_{4}\right)=0 .
\end{gathered}
$$

Note that both constraints are linear. The displacement of the beam under the clamping condition must minimize the energy $E$ while satisfying the constraints above. We rewrite $E(2.1)$ in terms of $\Delta \theta_{i}$ :

$$
E=\frac{1}{2} K \Delta \theta_{1}^{2}+\sum_{j=2}^{7} \frac{1}{2} K\left(\Delta \theta_{j}-\Delta \theta_{j-1}\right)^{2} .
$$

To minimize $E$ subject to (3.6a) and (3.6b), we use the Lagrange multiplier method. Define the Lagrangian

$$
L=E+\lambda_{1} c_{1}^{T} \Delta \boldsymbol{\theta}+\lambda_{2} c_{2}^{T} \Delta \boldsymbol{\theta}
$$

so that the first-order necessary condition is

$$
\left[\begin{array}{ccccc|cc}
2 K & -K & & & & & \\
-K & 2 K & -K & & & & \\
& \ddots & \ddots & \ddots & & c_{1} & c_{2} \\
& & -K & 2 K & -K & & \\
& & & -K & K & & \\
\hline & & c_{1}^{T} & & & 0 & 0 \\
& & c_{2}^{T} & & & 0
\end{array}\right]\left[\begin{array}{c} 
\\
\bar{\theta} \\
\overline{\lambda_{1}} \\
\lambda_{2}
\end{array}\right]=\left[\begin{array}{c} 
\\
\overline{0} \\
\\
\theta_{\mathrm{fix}}-\phi_{4}
\end{array}\right] .
$$


Using standard techniques from optimization, the first-order conditions (3.9) determine the critical points of the Lagrangian. To further determine which critical points correspond to actual constrained minima, second-order information from the Hessian or, in the constrained setting, the bordered Hessian, is usually required. Since the matrix in the upper left part of (3.9) is positive definite and the matrix $\left[c_{1} \mid c_{2}\right]$ is rank 2 , we apply a theorem from Luenberger $[5$, p. 424] to guarantee that the unique minimizer is given by the solution to the first-order conditions.

Solving this linear system allows us to find the desired displacement after clamping. Note that since $x_{i}$ does not change, we only need to keep track of $y_{i}$, given by (3.2b), once $\Delta \theta_{i}$ are found.

The main lesson that we draw from this calculation is that because the $x$-geometry is fixed, it does not enter the problem except to determine $t$ in the clamping. Once $t$ is determined, only the angle and $y$ constraints enter into the energy minimization. This simple behavior is precisely the reason for the model's lack of dependence on the order in which the welds are applied.

3.2. First Weld. Let us investigate welding under this model. To do so, we identify two beams with seven links by superscripts (1) and (2). The key is to track how link 4 of each beam maintains its relative position after welding. We note that all clamping has done is to put link 4 in each beam into position to be joined. The welding process that joins the beams is completely independent of the clamping process in that no parameters entering into the welding stage are determined by the clamping.

The relative position is maintained after joining if

$$
\begin{aligned}
& x_{4}^{(2)}=x_{4}^{(1)}+s \cos \theta_{4}^{(1)}, \\
& y_{4}^{(2)}=y_{4}^{(1)}+s \sin \theta_{4}^{(1)}, \\
& \theta_{4}^{(2)}=\theta_{4}^{(1)},
\end{aligned}
$$

where $s$ represents the signed distance along link 4 of beam 1 . In section 4 , for the sequence-dependent case, we will substitute the angles and coordinates of node 4 obtained from clamping into the above to solve for $s$. However, recall that in this model, the $x$-coordinate of the nodes are fixed by the initial angles of the links. Therefore, $s$ must be determined from (3.10a) only. This gives a formula for $s$ :

$$
s \approx \frac{x_{4}^{(2)}-x_{4}^{(1)}}{\cos \phi_{4}^{(1)}}=\frac{\sum_{j=1}^{3}\left(\cos \phi_{j}^{(2)}-\cos \phi_{j}^{(1)}\right)}{\cos \phi_{4}^{(1)}} .
$$

The constraints (3.10b) and (3.10c) are now applied. They simplify to

$$
\begin{gathered}
\sum_{j=1}^{3}\left(\Delta \theta_{j}^{(2)}-\Delta \theta_{j}^{(1)}\right)-s \Delta \theta_{4}^{(1)}=s \sin \phi_{4}^{(1)}+\sum_{j=1}^{3}\left(\sin \phi_{j}^{(1)}-\sin \phi_{j}^{(2)}\right), \\
\Delta \theta_{4}^{(2)}-\Delta \theta_{4}^{(1)}=\phi_{4}^{(1)}-\phi_{4}^{(2)} .
\end{gathered}
$$

Now, to find the positions of the beams after welding, we need to minimize $E$ in (3.7) subject to constraints (3.12a) and (3.12b). In the same manner described in section 3.1 , we write the constraints as vector equations

$$
\begin{gathered}
d_{1}^{T} \Delta \boldsymbol{\theta}^{1,2}-G=0, \\
d_{2}^{T} \Delta \boldsymbol{\theta}-\left(\phi_{4}^{1}-\phi_{4}^{2}\right)=0,
\end{gathered}
$$


where

$$
\begin{gathered}
d_{1}=(-1,-1,-1,-s, 0,0,0,1,1,1,0,0,0,0), \\
d_{2}=(0,0,0,-1,0,0,0,0,0,0,1,0,0,0), \\
G=s \sin \phi_{4}^{(1)}+\sum_{j=1}^{3}\left(\sin \phi_{j}^{(1)}-\sin \phi_{j}^{(2)}\right),
\end{gathered}
$$

and $\Delta \boldsymbol{\theta}^{1,2}=\left(\Delta \theta^{(1)}, \Delta \theta^{(2)}\right)$. The Lagrangian is

$$
L=E^{1}+E^{2}+\lambda_{1} d_{1}^{T} \Delta \boldsymbol{\theta}^{1,2}+\lambda_{2} d_{2}^{T} \Delta \boldsymbol{\theta}^{1,2},
$$

where $E^{1}$ and $E^{2}$ refer to expression (3.7) with $\Delta \boldsymbol{\theta}^{(1)}$ and $\Delta \boldsymbol{\theta}^{(2)}$, respectively. The corresponding first-order condition can be written in terms of a matrix equation for the vectors $\Delta \boldsymbol{\theta}^{(1)}$ and $\Delta \boldsymbol{\theta}^{(2)}$ :

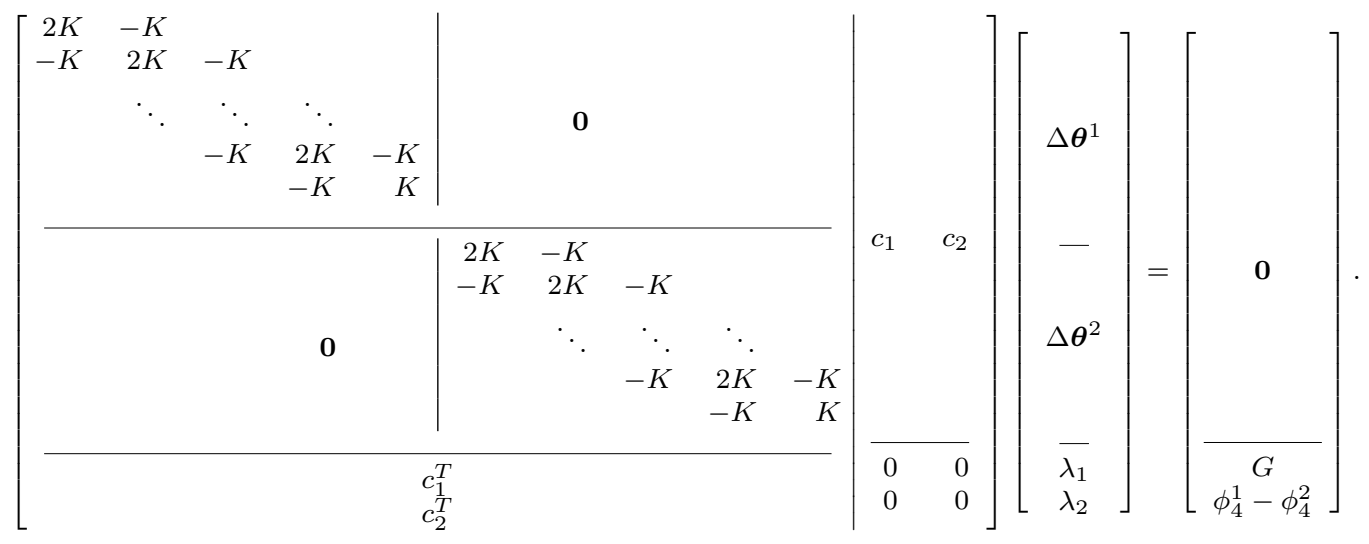

3.3. Second Weld. Suppose we take the joined beams and apply a second clamp along link 7 of each beam. The equations representing this weld are

$$
\begin{aligned}
& x_{7}^{(2)}=x_{7}^{(1)}+r \cos \theta_{7}^{(1)}, \\
& y_{7}^{(2)}=y_{7}^{(1)}+r \sin \theta_{7}^{(1)}, \\
& \theta_{7}^{(2)}=\theta_{7}^{(1)},
\end{aligned}
$$

where now the parameter $r$ represents the signed distance along link 7 . We note that these equations have the same form as (3.10a)-(3.10c) for the weld along link 4, and the parameter $r$ is analogous to the parameter $s$. As with the case with the weld along link 4, the initial geometry of the beams under this model determines $r$ by means of (3.14a). Equations (3.14b)-(3.14c) maintain the proper relative positions of link 7 and again translate to two linear constraints on the vectors $\Delta \boldsymbol{\theta}^{(1)}$ and $\Delta \boldsymbol{\theta}^{(2)}$ :

$$
\begin{gathered}
\sum_{j=1}^{6}\left(\Delta \theta_{j}^{(2)}-\Delta \theta_{j}^{(1)}\right)-r \Delta \theta_{7}^{(1)}=r \sin \phi_{7}^{(1)}+\sum_{j=1}^{6}\left(\sin \phi_{j}^{(1)}-\sin \phi_{j}^{(2)}\right), \\
\Delta \theta_{7}^{(2)}-\Delta \theta_{7}^{(1)}=\phi_{7}^{(1)}-\phi_{7}^{(2)} .
\end{gathered}
$$

The positions of the joined beams with these two welds are now found by minimizing $E$ subject to linear constraints (3.12a)-(3.12b) and (3.15)-(3.16). 
3.4. Sequence Independence. That the welded product is independent of whichever weld is applied first can be seen by the fact that both parameters $s$ and $r$ in the constraints are determined solely from the initial geometry of the two beams. Therefore, the resulting displacement is totally insensitive to the order in which the clamping and welding is done. We can conclude that this simple model is inadequate to explain the observed phenomenon of sequence dependence.

4. Model with Sequence Dependence. To make the previous model truly nonlinear, and hence provide the desired attribute of sequence dependence, we remove assumption (A). This means that the coordinates of the nodes are given by (3.1). By removing this assumption, the constraints involving the positions of the nodes will become nonlinear. Moreover, the parameters $s$ and $r$, which determine the relative positions of the joined links, are found by solving first for the clamped configurations. Clearly, this nonlinear dependence on the clamping configuration, which in turn depends nonlinearly on the first weld, suggests why this model will be sequence dependent. We also remark that unlike the model that did not demonstrate any sequence dependence, we cannot solve this model subject to either the clamping or the welding conditions analytically. As a consequence, the results that we present here are the numerical results obtained by the constr function in MATLAB. This routine solves optimization problems with equality and inequality constraints (see also fmincon).

4.I. Clamping. Consider again the beam consisting of seven links as shown in Figure 3. Recall that once the clamp is applied, a point on the workpiece will be held at $\left(x_{\mathrm{fix}}, y_{\mathrm{fix}}\right)$. Here, we remark that the key observation that led to the model of sequence dependence came from in-depth discussions about the exact clamping mechanism that is used. To envision the clamping procedure, consider a wire representing a beam between two "alligator jaws" representing the clamp. As the "jaws" or the clamp closes, the wire will slide laterally to accommodate the movement in the clamp. In the previous section, the $x$-coordinate of the nodes was fixed, which eliminated any possibility that the beam would slide during the clamping process. Here, as illustrated in Figure 4, the model allows the link that is being clamped, and hence the beam, to slide laterally during the clamping procedure. Once the clamp is closed the beam is held at $\left(x_{\mathrm{fix}}, y_{\mathrm{fix}}\right)$ at an angle of $\theta_{\mathrm{fix}}$. Although these conditions amount to three constraints,

$$
\begin{aligned}
& x_{4}=x_{\mathrm{fix}}+t \cos \theta_{4}, \\
& y_{4}=y_{\mathrm{fix}}+t \sin \theta_{4}, \\
& \theta_{4}=\theta_{\mathrm{fix}},
\end{aligned}
$$

similar to (3.3a)-(3.3c), we will not make any approximations to simplify them. Unlike the previous model, where the small angle and small displacement assumptions allowed us to use the linear equations (3.2) to determine the positions of the beams, here we must use (3.1), which is nonlinear, and hence we cannot determine $t$ from the initial displacement. Rather, $t$ becomes another variable to be determined by the minimization. Thus, in order to determine the clamped displacement for each beam, we need to minimize the energy in the beam (2.1) subject to the nonlinear constraints (4.1a)-(4.1c). In contrast to the previous model in which we minimized the energy (3.7) over $\Delta \boldsymbol{\theta}$, in this model we minimize the energy (2.1) over $\boldsymbol{\theta}$ and $t$.

To demonstrate that this model with nonlinear constraints exhibits the desired sequence dependence in the welding process, it is sufficient to consider two beams 


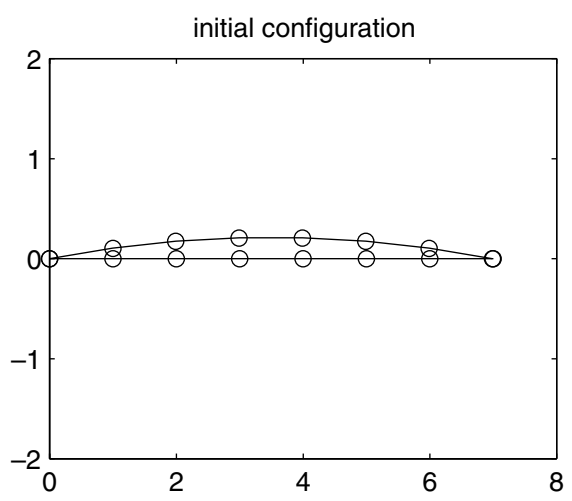

(a)

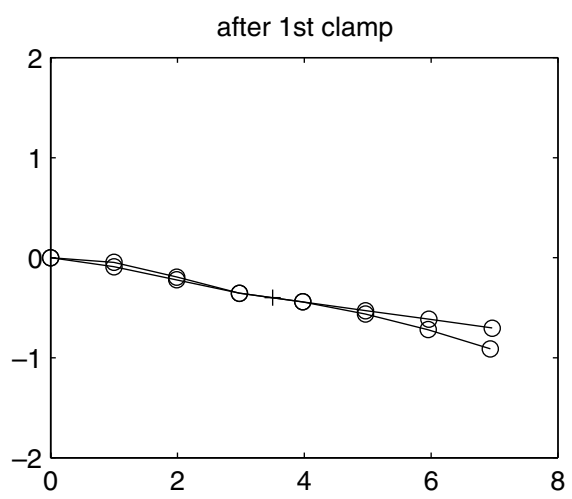

(b)

Fig. 4 (a) The original positions of the beams. (b) The beams after the fourth links are clamped at $(3.5,-0.4)$ at an angle of $-5^{\circ}$.

with randomly generated initial shape. We denote the initial angles for the first beam by $\theta_{j}^{(1)}$ and the initial angles for the second beam by $\theta_{j}^{(2)}, j=1: 7$. Similarly, the positions of the links for beam 1 are $\left(x_{j}^{(1)}, y_{j}^{(1)}\right)$, and those for beam 2 are $\left(x_{j}^{(2)}, y_{j}^{(2)}\right)$, $j=1$ : 8. The constraints in (4.1a)-(4.1c) will be applied to both beams. The configuration that corresponds to the minimum of the energy is computed numerically using the MATLAB function constr. Note that the energy is minimized over both $\boldsymbol{\theta}$ and $t$. The result of the calculation with $K=50, x_{\mathrm{fix}}=3.5, y_{\mathrm{fix}}=-0.4$, and $\theta_{\text {fix }}=-5^{\circ}$ is given in Figure 4 . The initial shapes of the beams are shown in Figure 4(a) and the resulting clamped configuration is shown in Figure 4(b). Notice that we have allowed the beams to pass through each other in their deformations, although a model that does not allow this to happen can be devised by adding inequality constraints. We do not consider this added complexity in the present work. Our goal is to demonstrate that sequence dependence is caused directly by the nonlinearity in the model.

4.2. First Weld. As in section 3.2 , we assume now that link 4 in each beam is going to be welded together. The clamped configuration implies a fixed relative position for link 4 of both beams. We model the weld as maintaining this relative position. This implies that the welding constraints depend on the configuration that resulted from clamping each beam. Therefore, if the position of node 4 in beam 1 and in beam 2 are

$$
\left(x_{4}^{(1)}, y_{4}^{(1)}\right) \text { and }\left(x_{4}^{(2)}, y_{4}^{(2)}\right)
$$

after clamping, then upon welding, the configuration must satisfy

$$
\begin{aligned}
& x_{4}^{(2)}=x_{4}^{(1)}+s \cos \theta_{4}^{(1)}, \\
& y_{4}^{(2)}=y_{4}^{(1)}+s \sin \theta_{4}^{(2)}, \\
& \theta_{4}^{(2)}=\theta_{4}^{(1)} .
\end{aligned}
$$

The value $s$ is determined by substituting the clamping results $\left(x_{4}^{(1)}, y_{4}^{(1)}\right)$ and $\left(x_{4}^{(2)}, y_{4}^{(2)}\right)$ in $(4.2 \mathrm{a})-(4.2 \mathrm{~b})$ and solving for $s$. The variable $s$ represents the signed distance between the fourth nodes in both beams upon clamping. 


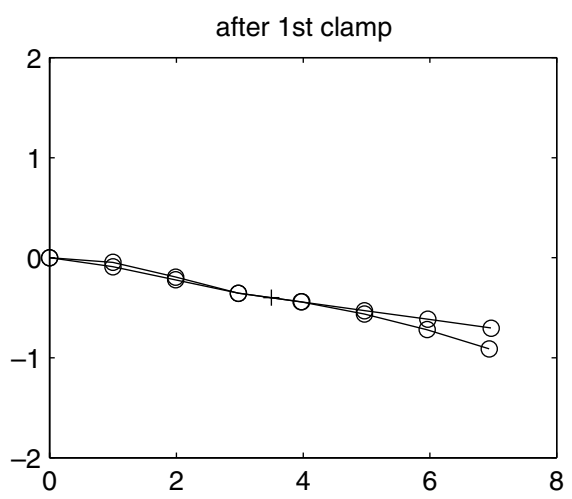

(a)

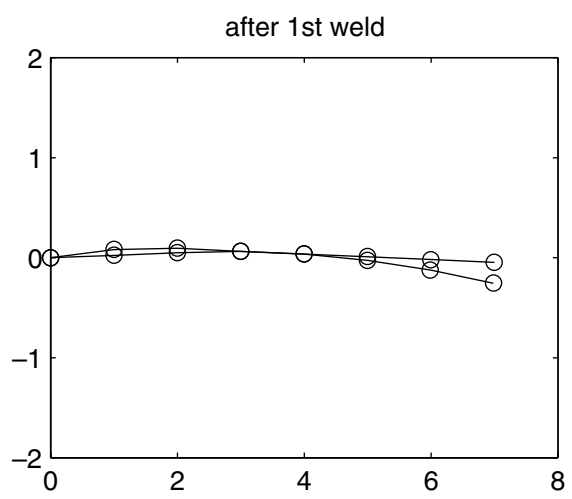

(b)

Fig. 5 (a) The beams after the fourth links are clamped at $(3.5,-0.4)$ at an angle of $-5^{\circ}$. (b) The beams after the first weld.

To determine the displacements in both beams after welding and releasing the clamp, we numerically minimize (2.1) over the angles $\theta_{j}^{(1)}$ and $\theta_{j}^{(2)}$ subject to the constraints (4.2a)-(4.2c) with the determined $s$. Figure 5 shows what happens to the clamped parts in Figure 4 after the fourth links are welded together and the clamp released. The key concept here is that clamping determines the relative position of the links before welding, and that welding maintains this relative position.

Note that when we solve for the clamped configurations, the constraints are uncoupled, and the displacement of the beams can be solved separately. When the beams are welded, the welding conditions $(4.2 \mathrm{a})-(4.2 \mathrm{c})$ couple the two beams and the configurations of the beams cannot be solved for independently.

4.3. Second Weld. Now we complete the joining process by applying clamps to the seventh links in both beams and then welding them together. Again, the clamping process will determine the relative position of the seventh links, while the final weld will retain this relative position.

As the beams are clamped, the constraints (4.2) implied by the weld in the link 4s still apply. Additional constraints are implied by the clamp, namely, a point in link 7 in each beam must end up in a specified position, and it must have a prescribed angle at that position. Without the risk of confusion, let that position be $\left(x_{\mathrm{fix}}, y_{\mathrm{fix}}\right)$ and the angle be $\theta_{\text {fix }}$. Then the new constraints, which apply to both beams, are

$$
\begin{aligned}
& x_{7}=x_{\mathrm{fix}}+t \cos \theta_{7}, \\
& y_{7}=y_{\mathrm{fix}}+t \sin \theta_{7}, \\
& \theta_{7}=\theta_{\mathrm{fix}},
\end{aligned}
$$

where again $t$ is unknown. Thus the second clamping problem is to find the angles $\theta_{j}^{(1)}$ and $\theta_{j}^{(2)}, j=1: 7$, and the value $t$ that minimizes the energy in (2.1) subject to constraints (4.2) and (4.3).

Having determined the new configuration of the beams after the second clamping, we have the positions of node 7 in both beams. Let us denote them by

$$
\left(x_{7}^{(1)}, y_{7}^{(1)}\right) \text { and }\left(x_{7}^{(2)}, y_{7}^{(2)}\right) .
$$




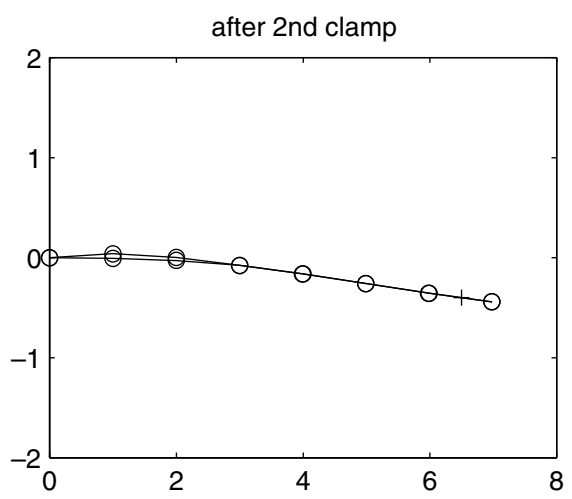

(a)

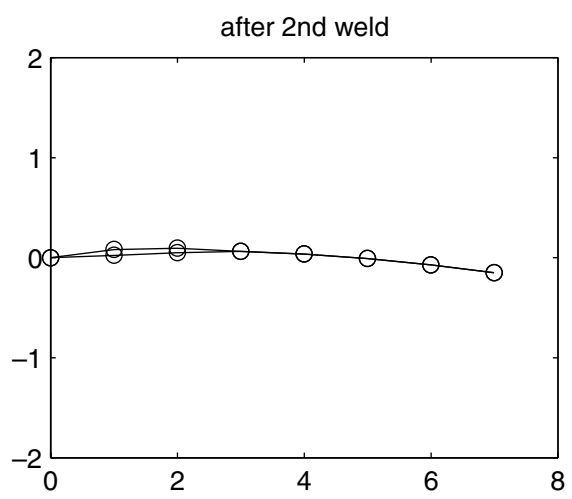

(b)

Fig. 6 (a) The beams after the seventh links are clamped at $(6.5,-0.4)$ at an angle of $-5^{\circ}$. (b) The beams after the second weld.

Then upon welding, the configuration must satisfy

$$
\begin{aligned}
& x_{7}^{(2)}=x_{7}^{(1)}+r \cos \theta_{7}^{(1)}, \\
& y_{7}^{(2)}=y_{7}^{(1)}+r \sin \theta_{7}^{(2)}, \\
& \theta_{7}^{(2)}=\theta_{7}^{(1)} .
\end{aligned}
$$

To determine $r$, we substitute the values of $\left(x_{7}^{(1)}, y_{7}^{(1)}\right)$ and $\left(x_{7}^{(2)}, y_{7}^{(2)}\right)$ obtained from the clamped configuration in the first two equations of (4.4) and solve for $r$. With $r$ found, we can find the final positions of the welded beams by minimizing energy in (2.1) subject to constraints (4.2) and (4.4). Figure 6 shows the result of first clamping at $(6.5,-0.4)$ at angle $-5^{\circ}$, and then welding link 7 .

Remark. Modeling more than two spot welds on a pair of beams can proceed in much the same way as we have described for the case of two welds. The important features of the model are that

1. clamping determines the relative positions of the links that are joined,

2. welding maintains the relative positions through subsequent deformations,

3. all previous welding constraints must be satisfied, particularly when new clamps and new welds are applied.

By following these simple rules, we can now reverse or alter any clamping/welding sequence and examine its effects.

4.4. Sequence Dependence. Since the welding constraints depend on the configuration of the clamped beams, this model demonstrates the desired sequence dependence. In contrast to the model in section 3 , where the $x$-coordinate of the beam was fixed by the linearity of the clamping constraints, the nonlinear model presented in this section allows the beam to slide during the clamping procedure, thus giving rise to different configurations depending upon the order in which the clamps and welds are applied.

To be more explicit, consider the resulting joined beams under two distinct clamping/welding sequences:

I: clamping first at $(3.5,-0.4)$ at $-5^{\circ}$, welding link 4 , and then clamping at $(6.5,-0.4)$ at $-5^{\circ}$, followed by welding link 7 ; 


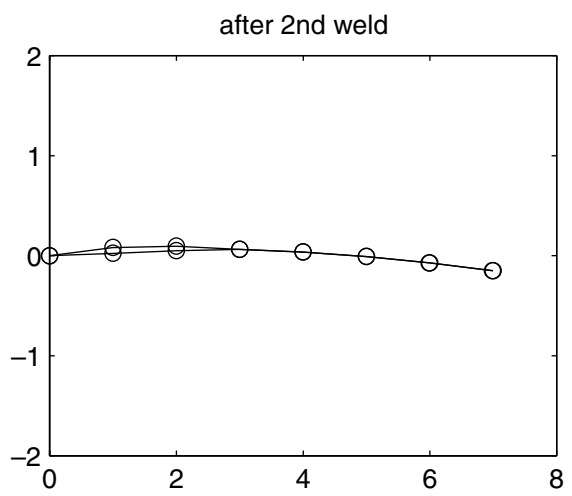

(a)

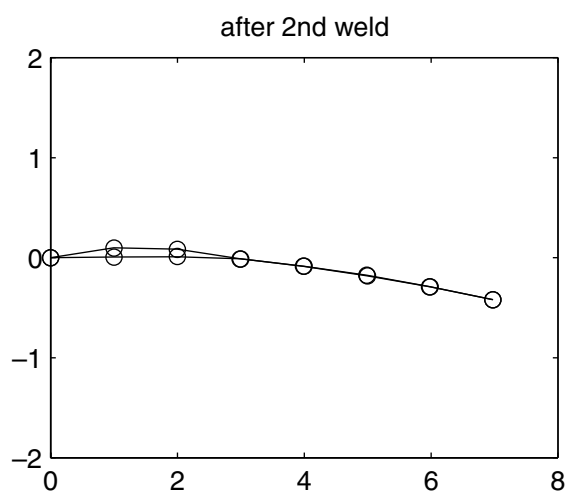

(b)

Fig. 7 (a) The resulting joined beams under sequence I. (b) The resulting joined beams under sequence II.

II: clamping first at $(6.5,-0.4)$ at $-5^{\circ}$, welding link 7 , and then clamping at $(3.5,-0.4)$ at $-5^{\circ}$, followed by welding link 4 .

In Figure 7 we show the results of sequence I in (a) and the results of sequence II in (b). The two welding sequences are shown side by side in Figure 8.

5. Statistical Simulations. We next perform a Monte Carlo simulation to determine the overall statistical properties of the joined products. The study attempts to find out if a certain weld sequence is better than another in the presence of variations in parts and clamping conditions.

To simulate the variation in parts, we assume that each of the angles $\phi_{j}^{(1)}$ and $\phi_{j}^{(2)}$, $j=1: 7$, are random with a normal distribution with a mean of $0^{\circ}$ and a standard deviation of $2^{\circ}$. That is, both beams are nominally flat. To model the variation in the clamp, we assume that while $x_{\mathrm{fix}}$ and $\theta_{\mathrm{fix}}$ are constants, $y_{\text {fix }}$ is a random variable with normal distribution with a mean of 0 and standard deviation of 0.2 . The standard deviation is to be compared with the size of the beam which stretches out to length 7. The quality of the product is measured by the end displacement of beam 2 , the idea being that if this displacement is small, the joined product is more or less flat.

Based on a thousand simulations we plot the variation of the final assembly as measured by the vertical position of the right end of beam 2. The results for the tooling sequence $l_{1}$ followed by $l_{2}$ can be seen in Figure 9 , and the results of the simulation for the tooling sequence $l_{2}$ followed by $l_{1}$ can be seen in Figure 10. Figure 9 has a mean of 0.0044 with standard deviation 0.0892 , whereas Figure 10 has a mean of 0.0043 and a standard deviation of 0.1305 . The means are comparable but the smaller standard deviation of the assembly with $l_{1}$ followed by $l_{2}$ distinguishes that method as the preferable one.

6. Conclusions. In this paper, we have presented a simple beam model that describes and explains how the final configuration of the beams is affected by the order in which a series of clamps and welds are applied. We first examined a model that assumes small initial angles and small displacements results in a beam whose $x$ displacement is zero. This led to linear constraints on the $y$-displacements and angle changes when modeling the clamping and welding. Such a model fails to exhibit the observed weld sequence dependence. A second model, where the $x$-displacements are 

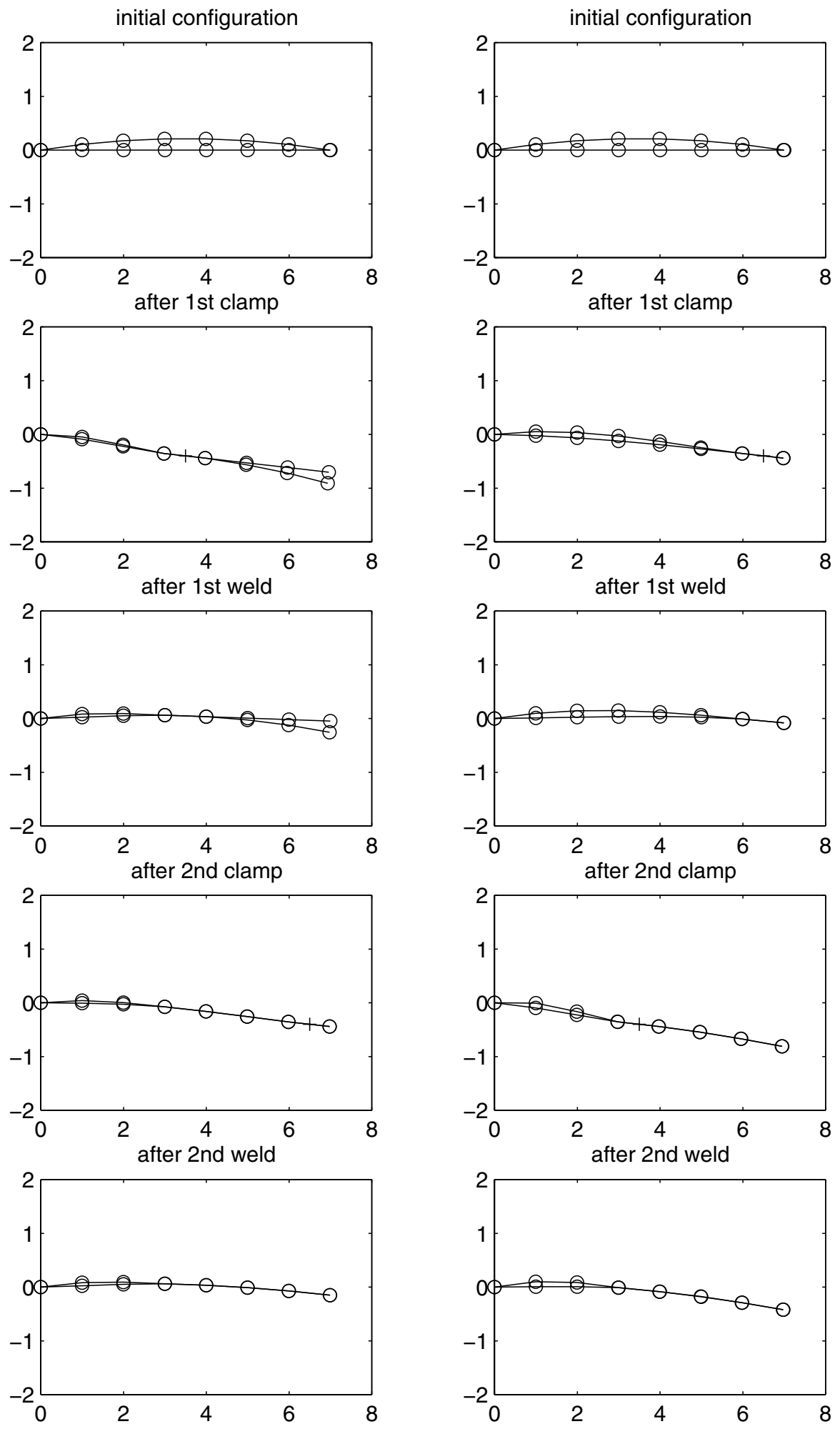

Fig. 8 Side-by-side comparison of the two joining sequences. 


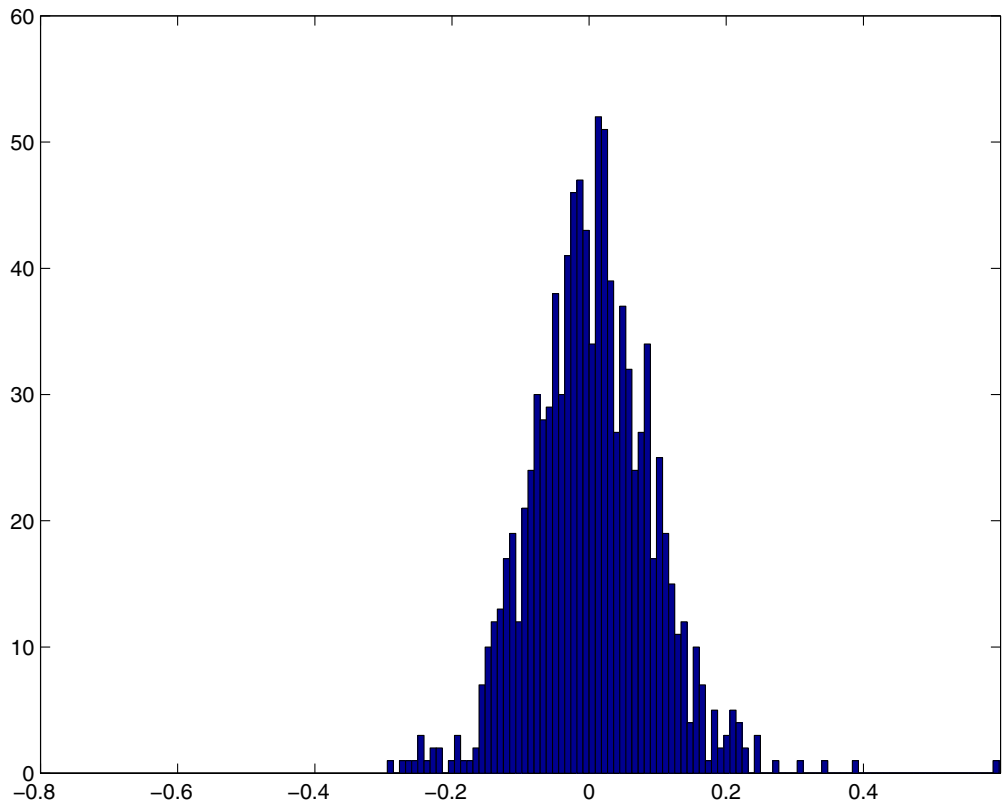

Fig. 9 Variation of the final assembly as measured by the vertical position of the second beam for the tooling sequence $l_{1}=\left(x_{1}, y_{1}\right)$ followed by $l_{2}=\left(x_{2}, y_{2}\right)$ with $x_{1}<x_{2}$.

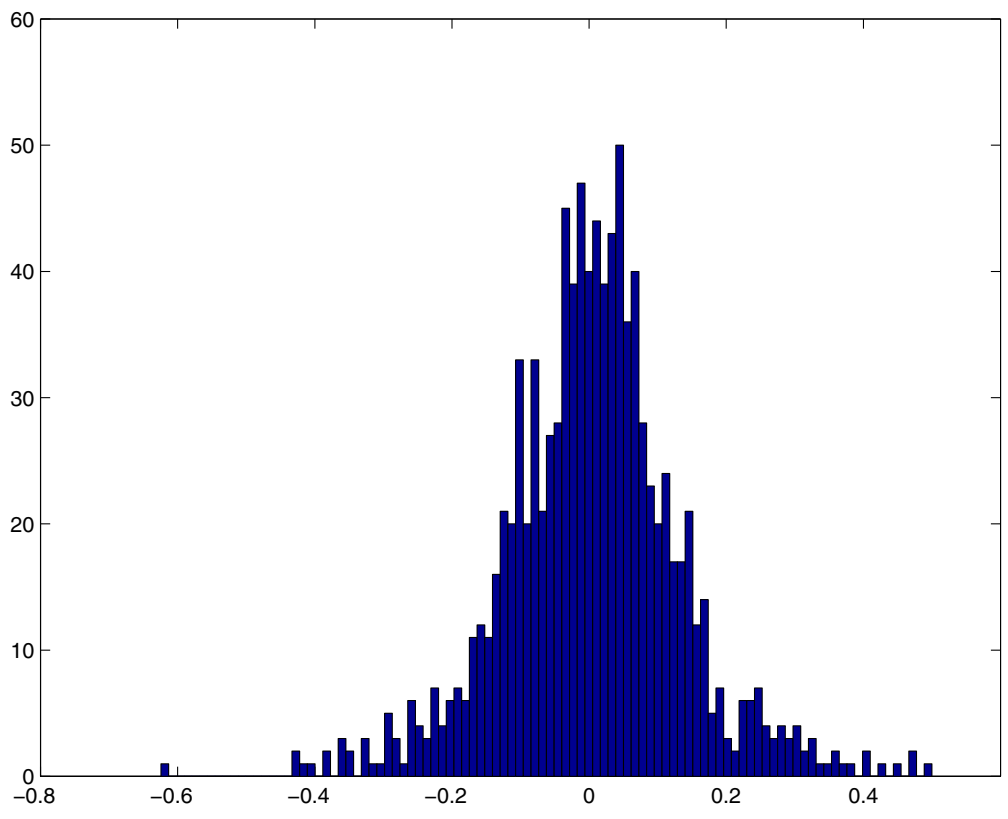

Fig. 10 Variation of the final assembly as measured by the vertical position of the second beam for the tooling sequence $l_{2}=\left(x_{2}, y_{2}\right)$ followed by $l_{1}=\left(x_{1}, y_{1}\right)$ with $x_{1}<x_{2}$. 
allowed, showed how clamping determines the relative positions of the links to be joined. The relative position is maintained after welding. This simple fact makes the subsequent clamp/weld operation "remember" its past, and the model therefore exhibited the desired sequence dependence.

A Monte Carlo simulation was performed to evaluate the behavior of the welding process under variation in initial shapes and clamping conditions. It was found that the distribution with the smallest standard deviation (in measured right-end displacement) preferable corresponds to clamping and welding the beams from the inside out. Thus, in order to adhere to the mandated tolerances on the final assembled product, it would be best to perform all clamping and welding from the inside out. Perhaps this result is not surprising if we recall that when we hang wallpaper, we must glue it to the wall from one end of the paper to the other.

\section{Exercises and Class Discussion.}

1. Explain how linearity is important in the solution of this problem. Give examples of other situations in which linearity plays an important role.

2. Explain the difficulties in formulating this problem with a continuous beam model rather than a series of rigid links.

3. Outline the necessary changes and complications that arise if this study is done for sheets of metal instead of one-dimensional beams.

4. In section 3.3, write down the matrix equation for $\Delta \boldsymbol{\theta}^{(1)}$ and $\Delta \boldsymbol{\theta}^{(2)}$. Explain why this system has a solution and why that solution is the unique minimizer.

5. How do the results of the simulations depend on the chosen standard deviations? What restrictions or assumptions are there on the standard deviation and mean?

6. Discuss the validity of saying that one simulation is "better" than another based on the distance between the ends of the beams. Can you provide a different way of measuring "better"? What are the advantages and disadvantages of using both methods?

Acknowledgments. It is our pleasure to thank Dr. Samuel Marin and Dr. Danny Baker of General Motors Research and Development Center for bringing the problem to our attention and for many helpful discussions.

\section{REFERENCES}

[1] S. Liu, Variation Simulation for Deformable Sheet Metal Assembly, Ph.D. thesis, University of Michigan, 1995.

[2] S. C. Liu, S. J. Hu, And T. C. Woo, Tolerance analysis for sheet metal assemblies, J. Mech. Design, 118 (1996), pp. 62-67.

[3] S. C. LiU AND S. JACK Hu, An offset finite element model and its applications in predicting sheet metal assembly variation, Int. J. Mach. Tools Manufact., 35 (1995), pp. 1545-1557.

[4] S. C. LiU, H.-W. LEE, AND S. J. Hu, Variation simulation for deformable sheet metal assemblies using mechanistic models, Trans. of NAMRI, 1996, pp. 235-241.

[5] D. Luenberger, Linear and Nonlinear Programming, 2nd ed., Addison-Wesley, Reading, MA, 1984.

[6] K. Washizu, Variational Methods in Elasticity and Plasticity, Pergamon Press, New York, 1982. 